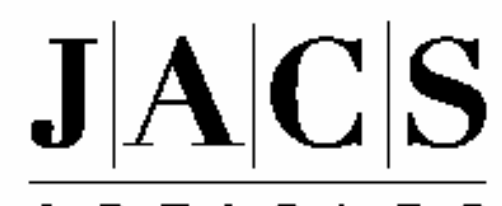

A R T I C L E S

\title{
Functionally Rigid Bistable [2]Rotaxanes
}

\author{
Sune Nygaard, ${ }^{\dagger}$ Ken C.-F. Leung, ${ }^{\ddagger}$ Ivan Aprahamian, ${ }^{\ddagger}$ Taichi Ikeda, ${ }^{\ddagger}$ Sourav Saha, ${ }^{\ddagger}$ \\ Bo W. Laursen, ${ }^{\S}$ Soo-Young Kim, ${ }^{\ddagger}$ Stinne W. Hansen, ${ }^{\dagger}$ Paul C. Stein, ${ }^{\dagger}$ Amar H. Flood, ${ }^{\ddagger}$ \\ J. Fraser Stoddart, ${ }^{, \neq}$and Jan O. Jeppesen ${ }^{\star, \dagger}$
}

Contribution from the Department of Chemistry, University of Southern Denmark (Odense University), Campusvej 55, DK-5230, Odense M, Denmark, California NanoSystems Institute and Department of Chemistry and Biochemistry, The University of California, Los Angeles, Los Angeles, 405 Hilgard Avenue, California 90095, USA and Department of Chemistry, University of Copenhagen, Universitetsparken 5, DK-2100, København Ø., Denmark

\section{Supporting Information}

* To whom correspondence may be addressed. E-mail: joj@chem.sdu.dk, stoddart@chem.ucla.edu

$\dagger$ Department of Chemistry, University of Southern Denmark (Odense University), Denmark

${ }^{\ddagger}$ Department of Chemistry and Biochemistry, The University of California, Los Angeles, California, USA

$\S$ Department of Chemistry, University of Copenhagen, Denmark 


\section{Contents}

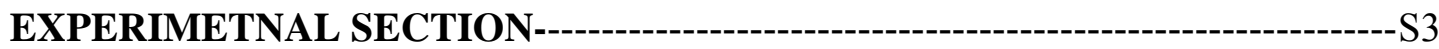

CHARACTERIZATION BY NMR SPECTROSCOPY-------------------------- S12

CHRACTERIZATION BY UV-VIS SPECTROSCOPY------------------- S12

Figure S1 Partial ${ }^{1} \mathrm{H}$ NMR spectrum $\left(500 \mathrm{MHz},\left(\mathrm{CD}_{3}\right)_{2} \mathrm{CO}\right)$ displaying------- S12 the aromatic region of $1.4 \mathrm{PF}_{6}$ at $198 \mathrm{~K}$

Figure S2 Partial ${ }^{1} \mathrm{H}$ NMR spectrum $\left(500 \mathrm{MHz},\left(\mathrm{CD}_{3}\right)_{2} \mathrm{CO}\right)$ displaying------- S13 the aliphatic region of $1.4 \mathrm{PF}_{6}$ at $198 \mathrm{~K}$

Figure S3 The full ${ }^{1} \mathrm{H}$ DQ COSY spectrum $\left(600 \mathrm{MHz},\left(\mathrm{CD}_{3}\right)_{2} \mathrm{CO}, 298 \mathrm{~K}\right)----\mathrm{S} 13$ that was used to assign the proton signals of $1 \bullet 4 \mathrm{PF}_{6}$

Figure S4 Partial ${ }^{1} \mathrm{H}$ DQ COSY spectrum $\left(600 \mathrm{MHz},\left(\mathrm{CD}_{3}\right)_{2} \mathrm{CO}\right)$ of----------- S14 $1.4 \mathrm{PF}_{6}$ at $298 \mathrm{~K}$

Figure S5 UV-vis spectra of 1.4PF 6 recorded in $\mathrm{MeCN}$ at various temperatures from 283 up to $323 \mathrm{~K}$

Figure S6 UV-vis spectra following the titrational addition of 1.0------------- S15 equivalent of $\mathrm{Fe}\left(\mathrm{ClO}_{4}\right)_{3}$ to a solution of $1.4 \mathrm{PF}_{6}$ in $\mathrm{MeCN}$

Figure S7 UV-vis spectra following the titrational addition of 1.0 ------------- S15 equivalent of $\mathrm{Fe}\left(\mathrm{ClO}_{4}\right)_{3}$ to a solution of $\mathbf{1}^{5+}$ in $\mathrm{MeCN}$

Figure S8 UV-vis spectra of the rigid MPTTF-NP dumbbell 18---------------- S16 upon oxidation

Table S1 Electrochemical Data for the Model Compounds ------------------ S16

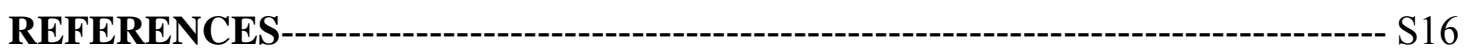

COMPLETE REFERENCES OF THE MAIN TEXT------------------------- S17 


\section{EXPERIMENTAL SECTION}

Materials. All reagents were purchased from Aldrich or Fluka, Inc. The starting materials 4,5-bis(bromomethyl)-1,3-dithiol-2-thione ${ }^{1}$ (3) 4-(2-cyanoethylthio)-1,3dithiole-2-thione ${ }^{2}$ (7), $\mathbf{9 a},{ }^{3} \mathbf{2 0} 2 \mathrm{PF}_{6},{ }^{4}$ were all prepared according to literature procedures. All reactions were carried out under an inert $\mathrm{N}_{2}$ or $\mathrm{Ar}$ atmosphere. $\mathrm{MeOH}$ was distilled from $\mathrm{Mg}$ while THF was distilled from Na-benzophenone prior to use. Triethylphosphite was distilled before use and was kept over molecular sieves (4 $\AA$ ).

General Methods. High-pressure reactions were carried out in a custom-made Teflon container under $10 \mathrm{kBar}$ of pressure generated by a Psika high-pressure apparatus. Melting points (mp) were determined either on an Electrothermal 9100 digital melting point apparatus or on a MEL-TEMP II melting point apparatus and are uncorrected. The 1D and 2D NMR spectra were recorded on a Varian $300 \mathrm{MHz}$, Bruker Avance 500 or Avance 600 pulsed FT spectrometers operating at 300, 500, and $600 \mathrm{MHz}$, respectively, for the recording of ${ }^{1} \mathrm{H}$ NMR spectra. Chemical shifts are quoted on the $\delta$ scale and coupling constants $(J)$ are expressed in Hertz $(\mathrm{Hz})$. Samples for ${ }^{1} \mathrm{H}$ NMR spectroscopic studies were prepared using solvents purchased from Cambridge Isotope Labs. All samples for kinetic analysis and oxidation/reduction studies were prepared in sealable $\mathrm{J}$. Young NMR tubes and were degassed using the freeze-pump-thaw method and then kept under argon. Temperatures were calibrated ${ }^{5}$ before or after each experiment with a neat $\mathrm{MeOH}$ sample and assumed to remain constant. All spectra were referenced using the residual solvent peak. The full assignment of the ${ }^{1} \mathrm{H}$ NMR signals was performed using COSY (correlation spectroscopy) and NOESY (nuclear overhauser effect spectroscopy) experiments. High resolution Fourier Transform matrix-assisted laser-desorption/ ionization mass spectrometry (HiResFT-MALDI-MS) was performed on an IonSpec 4.7 tesla Ultima Fourier Transform mass spectrometer, utilizing a 2,5-dihydroxybenzoic acid (DHB) matrix. Electrochemical and spectroelectrochemical experiments were carried out at room temperature in argon-purged $\mathrm{MeCN}$ or DMF solutions, with a Princeton Applied Research 263A Multipurpose instrument interfaced to a PC. Cyclic voltammetric experiments were performed using a glassy carbon working electrode $\left(0.018 \mathrm{~cm}^{2}\right.$, Cypress Systems). Its surface was polished routinely with a $0.05 \mu \mathrm{m}$ alumina-water slurry 
on a felt surface immediately before use. The counter electrode was a Pt wire and the reference electrode was an SCE one. Tetrabutylammonium hexafluorophosphate $\left(\mathrm{TBA} \cdot \mathrm{PF}_{6}\right)(0.1 \mathrm{M})$ was added as supporting electrolyte. The half-wave potential $\left(E_{1 / 2}\right)$ was calculated from an average of the cathodic and anodic cyclic voltammetric peaks. To establish the reversibility of a process, we used the criteria of (i) $60 \mathrm{mV}$ between cathodic and anodic peaks, and (ii) close to unity ratio of the intensities of the cathodic and anodic currents. Spectroelectrochemical experiments were made in a custom-built, opticallytransparent thin layer electrochemical (OTTLE) cell with an optical path of $1 \mathrm{~mm}$, using a Pt grid as working electrode, a Pt wire as counter electrode and a Ag wire pseudo reference electrode. Experimental errors: potential values, +/- $10 \mathrm{mV}$; absorption maxima, +/- $2 \mathrm{~nm}$. UV-vis spectroscopic data was recorded using a Cary 100 Bio spectrophotometer or a Shimadzu UV-1601PC apparatus. Elemental analyses were performed by Atlantic Microlabs, Inc., Atlanta, Georgia.

5-(4-Iodo-phenyl)-(1,3)dithiolo[4,5-c]pyrrole-2-thione (6). 4,5-Bis(bromomethyl)-1,3dithiol-2-thione (3) (3.84 g, $12.0 \mathrm{mmol})$ was dissolved in anhydrous THF:MeCN (1:2 v/v, $360 \mathrm{~mL}$ ). $\mathrm{K}_{2} \mathrm{CO}_{3}$ (5 eq., $60 \mathrm{mmol}, 8.3 \mathrm{~g}$ ) and p-iodoaniline (4) (1 eq. $12 \mathrm{mmol}, 2.8 \mathrm{~g}$ ) was added, whereafter the resulting unclear yellow solution was heated under reflux for $12 \mathrm{~h}$ or until the reaction was finished according to TLC analysis. After cooling to room temperature, the reaction mixture was filtered and the filter was washed with THF $(3 \times 50 \mathrm{~mL})$. Removal of the solvent from the combined organic phases gave the crude product 5-(4-iodo-phenyl)-4,6-dihydro-(1,3)dithiolo[4,5-c]pyrrole-2-thione (5) as a yellow powder. The yellow powder was suspended in anhydrous THF (150 mL), whereupon activated ${ }^{6} \mathrm{MnO}_{2}(25 \mathrm{~g})$ was added and the black reaction mixture was heated under reflux for $24 \mathrm{~h}$. The black reaction mixture was filtered through a short pad of Celite and the solvent was removed in vacuo. Addition of $\mathrm{H}_{2} \mathrm{O}(300 \mathrm{~mL})$ gave a precipitate, which was filtered affording the desired product 6 as a yellow solid ( $2.5 \mathrm{~g}$, $55 \%$ yield), mp $159-161{ }^{\circ} \mathrm{C} .{ }^{1} \mathrm{H}$ NMR $\left(\mathrm{CDCl}_{3}, 300 \mathrm{MHz}, 298 \mathrm{~K}\right): \delta=7.05$ (s, $\left.2 \mathrm{H}\right), 7.14$ (d, $J=8.8 \mathrm{~Hz}, 2 \mathrm{H}), 7.79$ (d, $J=8.8 \mathrm{~Hz}, 2 \mathrm{H})$; MS (EI): $m / z=375\left(M^{+}, 100 \%\right), 230$ (17), 203 (21), 172 (17), 76 (30); Elemental Analysis: calcd (\%) for $\mathrm{C}_{11} \mathrm{H}_{6} \mathrm{INS}_{3}$ : C 35.21, H 1.61, N 3.73, S 25.63; found C 35.48, H 1.89, N 3.81, S 25.34. 


\section{2-\{4-(2-Cyanoethylthio)-1,3-dithiole-2-yliden\}-5-(4-Iodo-phenyl)-(1,3)-}

dithiolo[4,5-c]pyrrole (8). The thione $6(375 \mathrm{mg}, 1.0 \mathrm{mmol})$ and the ketone 7 (205 $\mathrm{mg}$, $1.0 \mathrm{mmol})$ were suspended in distilled $(\mathrm{EtO})_{3} \mathrm{P}(15 \mathrm{~mL})$. The yellow brown suspension turned clear upon heating to $130{ }^{\circ} \mathrm{C}$. Additional ketone 7 (203 $\mathrm{mg}, 1.0 \mathrm{mmol}$ ) was added after $35 \mathrm{~min}$. The reaction mixture was stirred for an additional $1.5 \mathrm{~h}$. $\mathrm{MeOH}(15 \mathrm{~mL})$ was added after cooling to room temperature to precipitate the product $\mathbf{8}$ as a yellow powder. The resulting powder was washed with $\mathrm{MeOH}$ to give 8 as an analytically pure product (249 mg, 47\% yield), $\mathrm{mp}>220{ }^{\circ} \mathrm{C} .{ }^{1} \mathrm{H} \mathrm{NMR}\left(\mathrm{CD}_{3} \mathrm{SOCD}_{3}, 300 \mathrm{MHz}\right): \delta=2.85$ (t, $J=6.7 \mathrm{~Hz}, 2 \mathrm{H}), 3.08$ (t, $J=6.7 \mathrm{~Hz} 2 \mathrm{H}$,), 7.03 (s, $1 \mathrm{H}), 7.36$ (d, $J=8.8 \mathrm{~Hz}, 2 \mathrm{H}), 7.47$ $(\mathrm{s}, 2 \mathrm{H}), 7,79(\mathrm{~d}, J=8.8 \mathrm{~Hz}, 2 \mathrm{H}) ; \mathrm{MS}(\mathrm{MALDI}-\mathrm{TOF}): \mathrm{m} / \mathrm{z}=553\left(M^{+}+\mathrm{Na}\right), 530\left(M^{+}\right.$, $100 \%)$, HiRes-FT-MALDI-MS $m / z=529.8577\left(M^{+}, 100 \%\right)$, calcd for $\mathrm{C}_{17} \mathrm{H}_{11} \mathrm{IN}_{2} \mathrm{~S}_{5}: \mathrm{m} / \mathrm{z}$ $=529.8565$; Elemental Analysis: calcd (\%) for $\mathrm{C}_{17} \mathrm{H}_{11} \mathrm{IN}_{2} \mathrm{~S}_{5}$ : C 38.49, H 2.09, N 5.28; found C 38.10, H 2.13, N 5.28.

2-(2-\{4-[Bis(4-tert-butylphenyl)-(4-ethylphenyl)methyl]phenoxy\}ethoxy)ethyl Iodide (9).

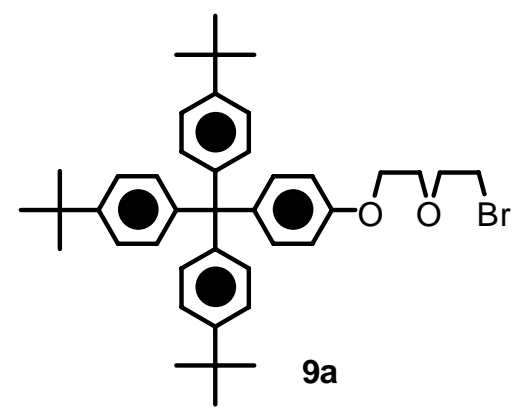

The bromide 9a $(1.31 \mathrm{~g}, 2.0 \mathrm{mmol})$ was dissolved in dry $\mathrm{Me}_{2} \mathrm{CO}(75 \mathrm{~mL})$ and $\mathrm{NaI}(4.5 \mathrm{~g}$, $30.0 \mathrm{mmol}$ ) was added. The clear colorless solution was heated under reflux for $2.5 \mathrm{~d}$ during which time the reaction mixture turned white and unclear. The solvent was then removed in vacuo, followed by redissolving of the white residue in $\mathrm{CH}_{2} \mathrm{Cl}_{2}(100 \mathrm{~mL})$ which was washed with a saturated aqueous solution of $\mathrm{Na}_{2} \mathrm{~S}_{2} \mathrm{O}_{4}(100 \mathrm{~mL}), \mathrm{H}_{2} \mathrm{O}$ $(2 \times 100 \mathrm{~mL})$, and then dried $\left(\mathrm{MgSO}_{4}\right)$. Evaporation of the solvent gave a white solid from which the product 9 was isolated by column chromatography $\left(\mathrm{SiO}_{2}\right.$, eluent: $\left.\mathrm{CH}_{2} \mathrm{Cl}_{2}\right)$ as a colorless band $\left(R_{\mathrm{f}}=0.5\right)$ that after evaporation gave 9 as a white solid (1.26 g, 90\%), 
mp 198-200 ${ }^{\circ} \mathrm{C} .{ }^{1} \mathrm{H} \mathrm{NMR}\left(\mathrm{CDCl}_{3}, 300 \mathrm{MHz}\right): \delta=1.30(\mathrm{~s}, 27 \mathrm{H}), 2.28(\mathrm{t}, J=6.5 \mathrm{~Hz}$, $2 \mathrm{H}), 3.71-3.87(\mathrm{~m}, 4 \mathrm{H}), 4.10(\mathrm{t}, J=6.5 \mathrm{~Hz}, 2 \mathrm{H}), 6.79$ (d, $J=9.0 \mathrm{~Hz}, 2 \mathrm{H}), 7.06-7.10$ $(\mathrm{m}, 8 \mathrm{H}), 7.21-7.25(\mathrm{~m}, 6 \mathrm{H}) ;{ }^{13} \mathrm{C} \mathrm{NMR}\left(\mathrm{CDCl}_{3}, 126 \mathrm{MHz}\right): \delta=2.7,31.4,63.1,67.3$, 69.4, 71.1, 113.1, 124.0, 130.7, 132.3, 139.9, 144.1, 148.3, 156.4; MS(MALDI): $\mathrm{m} / \mathrm{z}=$ $751\left(M^{+}+\mathrm{K}, 5\right), 725\left(M^{+}+\mathrm{Na}, 75\right), 569(100)$; HiRes-FT-MALDI - MS $\mathrm{m} / \mathrm{z}=725.2826$, calcd for $\mathrm{C}_{41} \mathrm{H}_{51} \mathrm{IO}_{2} \mathrm{Na} \mathrm{m} / \mathrm{z}=725.2847$; Elemental Analysis: calcd (\%) for $\mathrm{C}_{41} \mathrm{H}_{51} \mathrm{IO}_{2}: \mathrm{C}$ 70.07, H 7.31; found C 69.63, H 7.45.

Half-Dumbbell 10. Compound $8(60 \mathrm{mg}, 0.113 \mathrm{mmol})$ and the iodide 9 (120 mg, $0.170 \mathrm{mmol})$ were dissolved in anhydrous THF $(15 \mathrm{~mL})$. A solution of $\mathrm{CsOH} \cdot \mathrm{H}_{2} \mathrm{O}$ $(21 \mathrm{mg}, 0.125 \mathrm{mmol})$ in anhydrous $\mathrm{MeOH}(3 \mathrm{~mL})$ was added dropwise via a syringe. The reaction mixture was left to stir for $24 \mathrm{~h}$, causing the initial clear yellow solution to turn green and cloudy. The solvent was then removed in vacuo, yielding a yellow solid that was redissolved in $\mathrm{CH}_{2} \mathrm{Cl}_{2}(100 \mathrm{~mL})$, washed with $\mathrm{H}_{2} \mathrm{O}(3 \times 100 \mathrm{~mL})$, and then dried $\left(\mathrm{MgSO}_{4}\right)$. Column chromatography $\left(\mathrm{SiO}_{2}\right.$, eluent: $1: 1 \mathrm{v} / \mathrm{v}$ cyclohexane: $\left.\mathrm{CH}_{2} \mathrm{Cl}_{2}\right)$ gave the desired MPTTF half-dumbbell 10 as a yellow solid (62 mg, 52\% yield) after collection of a broad yellow band, mp 248-249 ${ }^{\circ} \mathrm{C} .{ }^{1} \mathrm{H}$ NMR $\left(\mathrm{CDCl}_{3}, 300 \mathrm{MHz}\right): \delta=1.29(\mathrm{~s}, 27 \mathrm{H})$, $2.96(\mathrm{t}, J=6.5 \mathrm{~Hz}, 2 \mathrm{H}), 3.75(\mathrm{t}, J=4.8 \mathrm{~Hz}, 2 \mathrm{H}), 3.82(\mathrm{t}, J=4.8 \mathrm{~Hz}, 2 \mathrm{H}), 4.10$ (t, $J=$ $6.5 \mathrm{~Hz}, 2 \mathrm{H}), 6.40$ (s, $1 \mathrm{H}), 6.75-6.79$ (m, $4 \mathrm{H}), 7.00-7.09$ (m, $12 \mathrm{H}), 7.20-7.24$ (m, $4 \mathrm{H})$, $7.70(\mathrm{~d}, J=8.8 \mathrm{~Hz}, 2 \mathrm{H}) ;{ }^{13} \mathrm{C} \mathrm{NMR}\left(\mathrm{CD}_{2} \mathrm{Cl}_{2}, 126 \mathrm{MHz}\right): 30.9,34.0,35.2,62.9,67.2$, $69.4,69.6,89.3,110.4,113.1,115.5,116.8,121.4,122.7,122.8,124.1,126.0,130.3$, $131.8,137.2,138.6,139.7,139.8,144.3,146.3,148.2,156.4$; MS(MALDI): $m / z=1074$ $\left([M+\mathrm{Na}]^{+}, \quad 10 \%\right), 1051\left(M^{+}, \quad 100 \%\right), 925\left([M-\mathrm{I}]^{+}, \quad 30 \%\right) ; \quad$ HiRes-FT-MALDI-MS $m / z=1051.2108\left(M^{+}, 100 \%\right)$, calcd for $\mathrm{C}_{55} \mathrm{H}_{58} \mathrm{INO}_{2} \mathrm{~S}_{5}: \mathrm{m} / \mathrm{z}=1051.2110$; Elemental Analysis: calcd (\%) for $\mathrm{C}_{55} \mathrm{H}_{58} \mathrm{INO}_{2} \mathrm{~S}_{5}$ : C 62.78, H 5.56, N 1.33; found C 63.39, H 5.67, N 1.33 .

5-Iodonaphthalen-1-ol (12). A solution of $\mathrm{NaNO}_{2}(2.2 \mathrm{~g}, 31 \mathrm{mmol})$ in $\mathrm{H}_{2} \mathrm{O}(8 \mathrm{~mL})$ was added dropwise to a solution of 5-aminonaphthalen-1-ol (11) $(5.0 \mathrm{~g}, 31 \mathrm{mmol})$ in $\mathrm{H}_{2} \mathrm{SO}_{4} / \mathrm{H}_{2} \mathrm{O}(5: 33 \mathrm{v} / \mathrm{v})$ at $0{ }^{\circ} \mathrm{C}$ over $1 \mathrm{~h}$. The mixture was then stirred for $15 \mathrm{~min}$, whereafter it was poured into a solution of $\mathrm{KI}(6.3 \mathrm{~g}, 38 \mathrm{mmol})$ in $\mathrm{H}_{2} \mathrm{O}(7 \mathrm{~mL})$ at $0{ }^{\circ} \mathrm{C}$. 
Subsequently, the resulting mixture was stirred at $60{ }^{\circ} \mathrm{C}$ until no gas was evolved. After that, the mixture was filtered and the solid collected, redissoved in $\mathrm{CH}_{2} \mathrm{Cl}_{2}$, and then washed successively with an aqueous solution of sodium metabisulfide ( $2 \mathrm{M})$ and brine, followed by drying $\left(\mathrm{MgSO}_{4}\right)$ and filtering. The filtrate was then concentrated under reduced pressure and the residue was subject to column chromatography $\left(\mathrm{SiO}_{2}\right)$ with hexanes/EtOAc (4:1) as the eluent to give the product 12 (3.4 g, 40\% yield) as a brown solid, mp 133.5-135.2. ${ }^{1} \mathrm{H}$ NMR $\left(\mathrm{CDCl}_{3}, 500 \mathrm{MHz}\right): \delta=5.26(\mathrm{~s}, 1 \mathrm{H}), 6.87(\mathrm{~d}, J=7.6$ $\mathrm{Hz}, 1 \mathrm{H}), 7.17$ (t, $J=7.6 \mathrm{~Hz}, 1 \mathrm{H}), 7.40$ (t, $J=7.6 \mathrm{~Hz}, 1 \mathrm{H}), 7.69$ (d, $J=8.4 \mathrm{~Hz}, 1 \mathrm{H})$, 8.10 (d, $J=7.6 \mathrm{~Hz}, 1 \mathrm{H}), 8.23$ (d, $J=8.4 \mathrm{~Hz}, 1 \mathrm{H})$; MS(EI): $m / z=269.9540\left(M^{+}, 100 \%\right)$; calcd for $\mathrm{C}_{10} \mathrm{H}_{7} \mathrm{IO}: \mathrm{m} / \mathrm{z}=269.9542$.

2-(2-(2,6-Diisopropylphenoxy)ethoxy)ethanol (14). A mixture of 2,6-diisopropylphenol (13) $(5.0 \mathrm{~g}, 28 \mathrm{mmol}), 2$-(2-chloroethoxy)ethanol (5.3 g, $42 \mathrm{mmol}), \mathrm{K}_{2} \mathrm{CO}_{3}(11.6 \mathrm{~g}$, $84 \mathrm{mmol})$ and $\mathrm{LiBr}(50 \mathrm{mg})$ in DMF $(80 \mathrm{~mL})$ was heated under reflux and stirred for $2 \mathrm{~d}$. The resulting mixture was cooled down to room temperature and filtered. The filtrate was concentrated and the residue was purified by column chromatography $\left(\mathrm{SiO}_{2}\right)$ with hexanes/EtOAc $(2: 1 \mathrm{v} / \mathrm{v})$ as the eluent to afford the product $14(4.8 \mathrm{~g}, 65 \%$ yield $)$ as a colorless liquid. ${ }^{1} \mathrm{H}$ NMR $\left(\mathrm{CDCl}_{3}, 500 \mathrm{MHz}\right): \delta=1.22$ (s, $\left.12 \mathrm{H}\right), 2.03$ (bs, $\left.1 \mathrm{H}\right), 3.38$ (sep, $J=6.9 \mathrm{~Hz}, 2 \mathrm{H}), 3.72(\mathrm{t}, J=4.8 \mathrm{~Hz}, 2 \mathrm{H}), 3.81(\mathrm{t}, J=4.8 \mathrm{~Hz}, 2 \mathrm{H}), 3.89$ (t, $J=4.8$ $\mathrm{Hz}, 2 \mathrm{H}), 3.93$ (t, $J=4.8 \mathrm{~Hz}, 2 \mathrm{H}), 7.09-7.20$ (brs, $3 \mathrm{H}) ;{ }^{13} \mathrm{C} \mathrm{NMR}\left(\mathrm{CDCl}_{3}, 126 \mathrm{MHz}\right)$ : $\delta=23.8,26.2,61.9,70.5,72.5,73.7,124.0,124.7,141.7,152.7$; MS(HiResMALDI) $m / z=289.1769\left([M+N a]^{+}, 100 \%\right)$, calcd for $\mathrm{C}_{16} \mathrm{H}_{26} \mathrm{O}_{3} \mathrm{Na}: \mathrm{m} / \mathrm{z}=289.1780$.

\section{1-(2-(2-(2,6-Diisopropylphenoxy)ethoxy)ethoxy)-5-iodonaphthalene}

(15). DIAD $(1.1 \mathrm{~mL}, 5.6 \mathrm{mmol})$ was added dropwise to a solution of $\mathrm{PPh}_{3}(1.5 \mathrm{~g}, 5.6 \mathrm{mmol})$ in $\mathrm{THF}$ $(15 \mathrm{~mL})$ at $0{ }^{\circ} \mathrm{C}$ in a degassed flask. The resulting mixture was then stirred for $15 \mathrm{~min}$ at $0{ }^{\circ} \mathrm{C}$. Subsequently, a mixture of 5-iodonaphthalen-1-ol $12(1.0 \mathrm{~g}, 3.7 \mathrm{mmol})$ and alcohol $14(1.0 \mathrm{~g}, 3.7 \mathrm{mmol})$ in THF $(15 \mathrm{~mL})$ was added dropwise to the mixture at $0{ }^{\circ} \mathrm{C}$ and then stirred for $18 \mathrm{~h}$ at $25^{\circ} \mathrm{C}$. After that, the mixture was concentrated under reduced pressure and the residue was purified by column chromatography $\left(\mathrm{SiO}_{2}\right)$ with hexanes/EtOAc $(5: 1 \mathrm{v} / \mathrm{v})$ as the eluent to give the product 15 (1.4 g, 73\% yield) as a brown oil. ${ }^{1} \mathrm{H}$ NMR 
$\left(\mathrm{CDCl}_{3}, 500 \mathrm{MHz}\right): \delta=1.23(\mathrm{~s}, 12 \mathrm{H}), 3.41(\mathrm{sep}, J=6.8 \mathrm{~Hz}, 2 \mathrm{H}), 3.90-4.02(\mathrm{~m}, 4 \mathrm{H})$, $4.12(\mathrm{t}, J=4.7 \mathrm{~Hz}, 2 \mathrm{H}), 4.38(\mathrm{t}, J=4.7 \mathrm{~Hz}, 2 \mathrm{H}), 6.92$ (d, $J=7.7 \mathrm{~Hz}, 1 \mathrm{H}), 6.91-6.95$ (brs, $3 \mathrm{H}), 7.15$ (t, $J=7.7 \mathrm{~Hz}, 1 \mathrm{H}), 7.47$ (t, $J=7.3 \mathrm{~Hz}, 1 \mathrm{H}), 7.69$ (d, $J=8.6 \mathrm{~Hz}, 1 \mathrm{H})$, $8.09(\mathrm{~d}, J=7.3,1 \mathrm{H}), 8.36(\mathrm{~d}, J=8.6 \mathrm{~Hz}, 1 \mathrm{H}) ;{ }^{13} \mathrm{C} \mathrm{NMR}\left(\mathrm{CDCl}_{3}, 126 \mathrm{MHz}\right): \delta=24.0$, $26.2,68.2,69.9,70.8,73.9,98.8,105.5,123.0,123.9,124.56,124.59,126.0,126.3$, 127.3, 135.2, 138.0, 141.7, 152.9, 154.4; MS(HiResMALDI): $m / z=541.1195\left([M+N a]^{+}\right.$, $100 \%$ ), calcd for $\mathrm{C}_{26} \mathrm{H}_{31} \mathrm{IO}_{3} \mathrm{Na}: \mathrm{m} / \mathrm{z}=541.1210$.

((5 - (2 - (2 - (2,6 - Diisopropylphenoxy) ethoxy) ethoxy) naphthalenyl) ethynyl) trimethylsilane (16). A degassed mixture of iodide 15 (0.49 g, $0.95 \mathrm{mmol})$, trimethylsilylacetylene $(1.4 \mathrm{~mL}, 9.5 \mathrm{mmol}),\left(\mathrm{PPh}_{3}\right)_{2} \mathrm{PdCl}_{2}(0.14 \mathrm{~g}, 0.19 \mathrm{mmol}), \mathrm{CuI}$ (18 mg, $0.095 \mathrm{mmol}), \mathrm{PPh}_{3}(25 \mathrm{mg}, 0.095 \mathrm{mmol})$ and $\mathrm{Et}_{3} \mathrm{~N}$ (1.3 mL, 9.5 mmol) in $\mathrm{PhMe}$ $(10 \mathrm{~mL})$ was stirred at $60^{\circ} \mathrm{C}$ for $1 \mathrm{~d}$. The mixture was then filtered through a short pad of Celite. The filtrate collected was concentrated under reduced pressure and the residue was purified by column chromatography $\left(\mathrm{SiO}_{2}\right)$ using a gradient of hexanes to hexanes/EtOAc $(8: 1 \mathrm{v} / \mathrm{v})$ to give the product $16(0.41 \mathrm{~g}, 90 \%$ yield $)$ as a brown solid, $\mathrm{mp}$ 71.8-74.3 ${ }^{\circ} \mathrm{C} .{ }^{1} \mathrm{H}$ NMR $\left(\mathrm{CDCl}_{3}, 500 \mathrm{MHz}\right): \delta=0.33$ (s, $\left.9 \mathrm{H}\right), 1.21$ (s, $\left.12 \mathrm{H}\right), 3.41$ (sep, $J=6.9 \mathrm{~Hz}, 2 \mathrm{H}), 3.93-4.02(\mathrm{~m}, 4 \mathrm{H}), 4.11(\mathrm{t}, J=4.9 \mathrm{~Hz}, 2 \mathrm{H}), 4.37$ (t, $J=4.6 \mathrm{~Hz}, 2 \mathrm{H})$, $6.89(\mathrm{~d}, J=7.6 \mathrm{~Hz}, 1 \mathrm{H}), 7.06-7.12$ (brs, $3 \mathrm{H}), 7.38$ (t, $7.3 \mathrm{~Hz}, 1 \mathrm{H}), 7.47$ (t, $J=7.3 \mathrm{~Hz}$, $1 \mathrm{H}), 7.71(\mathrm{~d}, J=7.1 \mathrm{~Hz}, 1 \mathrm{H}), 7.93(\mathrm{~d}, J=8.4 \mathrm{~Hz}, 1 \mathrm{H}), 8.32(\mathrm{~d}, J=8.4 \mathrm{~Hz}, 1 \mathrm{H})$; ${ }^{13} \mathrm{C} \mathrm{NMR}\left(\mathrm{CDCl}_{3}, 126 \mathrm{MHz}\right): \delta=0.1,24.0,26.2,68.0,70.0,70.8,73.9,99.0,103.3$, 105.4, 118.6, 120.1, 123.1, 123.9, 124.3, 125.4, 126.7, 128.4, 131.3, 131.9, 134.5, 141.7, 152.9, 154.7; MS(HiResMALDI): $\mathrm{m} / \mathrm{z}=511.2654\left([\mathrm{M}+\mathrm{Na}]^{+}, 100 \%\right)$, calcd for $\mathrm{C}_{31} \mathrm{H}_{40} \mathrm{O}_{3} \mathrm{SiNa}: \mathrm{m} / \mathrm{z}=511.2639$.

Half-Dumbbell 17. A mixture of compound 16 (1.0 g, $2.1 \mathrm{mmol})$ and $\mathrm{K}_{2} \mathrm{CO}_{3}(1.4 \mathrm{~g}, 10$ $\mathrm{mmol})$ in $\mathrm{MeOH} / \mathrm{THF}(1: 1 \mathrm{v} / \mathrm{v}, 10 \mathrm{~mL})$ was stirred at $25{ }^{\circ} \mathrm{C}$ for $2 \mathrm{~h}$. Subsequently, $\mathrm{H}_{2} \mathrm{O}$ $(20 \mathrm{~mL})$ was added to the mixture to quench the reaction, followed by the extraction with EtOAc $(2 \times 20 \mathrm{~mL})$. The collected extracts were combined and washed with brine, dried $\left(\mathrm{MgSO}_{4}\right)$ and filtered. The filtrate was concentrated under reduced pressure and the residue was purified by column chromatography $\left(\mathrm{SiO}_{2}\right)$ using hexanes/EtOAc $(8: 1 \mathrm{v} / \mathrm{v})$ as 
the eluent to give the half-dumbbell $17(0.50 \mathrm{~g}, 60 \%$ yield $)$ as a reddish oil. ${ }^{1} \mathrm{H}$ NMR $\left(\mathrm{CDCl}_{3}, 500 \mathrm{MHz}\right): \delta=1.22(\mathrm{~s}, 12 \mathrm{H}), 3.40$ (sep, $\left.J=6.9 \mathrm{~Hz}, 2 \mathrm{H}\right), 3.46$ (s, $\left.1 \mathrm{H}\right), 3.60$ $4.02(\mathrm{~m}, 4 \mathrm{H}), 4.12(\mathrm{t}, J=4.7 \mathrm{~Hz}, 2 \mathrm{H}), 4.38(\mathrm{t}, J=4.7 \mathrm{~Hz}, 2 \mathrm{H}), 6.90(\mathrm{~d}, J=7.7 \mathrm{~Hz}, 1$ H), 7.06-7.12 (brs, $3 \mathrm{H}), 7.41$ (t, $J=8.3 \mathrm{~Hz}, 1 \mathrm{H}), 7.48$ (t, $J=8.3 \mathrm{~Hz}, 1 \mathrm{H}), 7.74$ (d, $J=$ $7.7 \mathrm{~Hz}, 1 \mathrm{H}), 7.96$ (d, $J=8.5 \mathrm{~Hz}, 1 \mathrm{H}), 8.36$ (d, $J=8.5 \mathrm{~Hz}, 1 \mathrm{H}) ;{ }^{13} \mathrm{C} \mathrm{NMR}\left(\mathrm{CDCl}_{3}, 126\right.$ MHz): $\delta=22.5,24.0,68.0,69.9,70.8,73.9,81.6,82.0,105.4,118.4,119.1,123.4,123.9$, 124.2, 124.5, 125.4, 126.8, 131.7, 134.5, 141.7, 152.9, 154.8; MS(HiResMALDI): $\mathrm{m} / \mathrm{z}=$ $439.2257\left([M+\mathrm{Na}]^{+}, 100 \%\right)$, calcd for $\mathrm{C}_{28} \mathrm{H}_{32} \mathrm{O}_{3} \mathrm{Na}: \mathrm{m} / \mathrm{z}=439.2244$.

MPTTF-NP Dumbbell 18. A degassed mixture of the half-dumbbell 10 (100 mg, 0.095 mmol), half-dumbbell 17 (48 mg, $0.11 \mathrm{mmol}),\left(\mathrm{PPh}_{3}\right)_{2} \mathrm{PdCl}_{2}$ (14 mg, $\left.0.019 \mathrm{mmol}\right), \mathrm{CuI}$ (1.8 mg, $0.0095 \mathrm{mmol}), \mathrm{PPh}_{3}(2.5 \mathrm{mg}, 0.0095 \mathrm{mmol})$, and $\mathrm{Et}_{3} \mathrm{~N}(0.14 \mathrm{~mL}, 0.95 \mathrm{mmol})$ in PhMe $(2 \mathrm{~mL})$ was stirred at $60{ }^{\circ} \mathrm{C}$ for $1 \mathrm{~d}$. The mixture was then filtered through a short pad of Celite. The filtrate was concentrated under reduced pressure and the residue was purified by column chromatography $\left(\mathrm{SiO}_{2}\right)$ using hexanes/EtOAc $(5: 1 \mathrm{v} / \mathrm{v})$ to give the MPTTF-NP dumbbell 18 (120 mg, 94\% yield) as a yellowish solid, mp $>200{ }^{\circ} \mathrm{C}$, ${ }^{1} \mathrm{H} \mathrm{NMR}\left(\mathrm{CDCl}_{3}, 500 \mathrm{MHz}\right): \delta=1.21(\mathrm{~d}, J=3.4 \mathrm{~Hz}, 12 \mathrm{H}), 1.30(\mathrm{~s}, 27 \mathrm{H}), 2.98(\mathrm{t}, J=$ $3.4 \mathrm{~Hz}, 2 \mathrm{H}), 3.40$ (hep, $J=3.4 \mathrm{~Hz}, 2 \mathrm{H}), 3.76$ (t, $J=3.4 \mathrm{~Hz}, 2 \mathrm{H}), 3.82-3.86$ (m, $2 \mathrm{H}$ ), 3.98-4.04 (m, 4 H), 4.10-4.18 (m, 4 H), 4.38-4.42 (m, 2 H), $6.41(\mathrm{~s}, 1 \mathrm{H}), 6.78(\mathrm{~d}, J=$ $8.6 \mathrm{~Hz}, 2 \mathrm{H}), 6.88(\mathrm{~s}, 2 \mathrm{H}), 6.94(\mathrm{~d}, J=8.3 \mathrm{~Hz}, 1 \mathrm{H}), 7.06-7.12(\mathrm{~m}, 11 \mathrm{H}), 7.18-7.24(\mathrm{~m}$, $6 \mathrm{H}), 7.30(\mathrm{~d}, J=8.3 \mathrm{~Hz}, 2 \mathrm{H}), 7.46(\mathrm{t}, J=8.3 \mathrm{~Hz}, 1 \mathrm{H}), 7.50$ (t, $J=8.3 \mathrm{~Hz}, 1 \mathrm{H}) ; 7.63-$ 7.68 (m, $2 \mathrm{H}), 7.80$ (d, $J=8.3 \mathrm{~Hz}, 1 \mathrm{H}), 8.01(\mathrm{~d}, J=8.3 \mathrm{~Hz}, 1 \mathrm{H}), 8.35$ (d, $J=8.3 \mathrm{~Hz}, 1$ $\mathrm{H}) ;{ }^{13} \mathrm{C} \mathrm{NMR}\left(\mathrm{CD}_{2} \mathrm{Cl}_{2}, 126 \mathrm{MHz}\right): \delta=23.7,26.1,31.2,34.3,35.3,63.0,67.3,68.1,69.5$, 69.7, 70.0, 70.9, 74.0, 92.7, 92.8, 105.5, 110.5, 113.2, 115.5, 116.9, 118.6, 119.2, 119.3, $122.8,123.5,124.0,124.1,124.6,125.5,126.0,126.9,130.2,131.8,137.3,138.7,139.8$, $141.7,141.8,144.3,147.7,148.3,153.0,154.9,156.7$; MS(MALDI-TOF): $\mathrm{m} / \mathrm{z}=1363$ $\left([M+\mathrm{Na}]^{+}, 5 \%\right), 1340\left(M^{+}, 100 \%\right), 1103(30 \%), 1090(25 \%), 1075(10 \%), 765(25 \%)$ : HiRes-FT-MALDI-MS m/z = 1339.5313, calcd for $\mathrm{C}_{83} \mathrm{H}_{89} \mathrm{NO}_{5} \mathrm{~S}_{5}: \mathrm{m} / \mathrm{z}=1339.5340$.

NP-NP Dumbbell 19. TMEDA $(0.070 \mathrm{~mL}, 0.48 \mathrm{mmol})$ was added dropwise to a slurry of $\mathrm{CuCl}(48 \mathrm{mg}, 0.48 \mathrm{mmol})$ in $\mathrm{CHCl}_{3}(0.5 \mathrm{~mL})$ at $25^{\circ} \mathrm{C}$ and the resulting mixture was 
stirred for $15 \mathrm{~min}$ under air ( $1 \mathrm{~atm})$ prior to the addition of the solution of half-dumbbell $17(100 \mathrm{mg}, 0.24 \mathrm{mmol})$ in $\mathrm{CHCl}_{3}(0.5 \mathrm{~mL})$ to the mixture. Subsequently, the mixture was stirred for $1 \mathrm{~h}$ at $25{ }^{\circ} \mathrm{C}$ under air (1 atm). Then, an aqueous solution of $\mathrm{NH}_{4} \mathrm{Cl}(2 \mathrm{M}$, $2 \mathrm{~mL}$ ) was added to the mixture to quench the reaction, followed by the extraction with $\mathrm{CHCl}_{3}(2 \times 5 \mathrm{~mL})$. The organic layers were combined, washed with brine, dried $\left(\mathrm{MgSO}_{4}\right)$ and filtered. The filtrate was concentrated and the residue was purified by column chromatography $\left(\mathrm{SiO}_{2}\right)$ with hexanes/EtOAc $(8: 1)$ as the eluent to give the NP-NP dumbbell 19 (76 mg, 76\% yield) as a reddish oil. ${ }^{1} \mathrm{H}$ NMR $\left(\mathrm{CDCl}_{3}, 500 \mathrm{MHz}\right) \delta=1.20$ $(\mathrm{d}, J=2.9 \mathrm{~Hz}, 24 \mathrm{H}), 3.41$ (hep, $J=3.5 \mathrm{~Hz}, 4 \mathrm{H}), 3.95-4.03(\mathrm{~m}, 8 \mathrm{H}), 4.10-4.13(\mathrm{~m}, 4$ H), 4.36-4.43 (m, 4 H), 6.93 (d, J=7.8 Hz, 2 H), 7.10 (brs, $6 \mathrm{H}), 7.43$ (t, $J=7.8 \mathrm{~Hz}, 2$ H), 7.51 (t, $J=7.8 \mathrm{~Hz}, 2 \mathrm{H}), 7.83(\mathrm{~d}, J=7.8 \mathrm{~Hz}, 2 \mathrm{H}), 8.01$ (d, $J=7.8 \mathrm{~Hz}, 2 \mathrm{H}), 8.39$ (d, $J=7.8 \mathrm{~Hz}, 2 \mathrm{H}) ;{ }^{13} \mathrm{C} \mathrm{NMR}\left(\mathrm{CDCl}_{3}, 126 \mathrm{MHz}\right): \delta=24.0,26.2,68.1,69.9,70.8,73.9$, $78.4,81.1,105.7,118.5,119.0,123.9,124.4,124.6,124.7,125.5,127.2,132.5,135.0$, 141.7, 152.9, 154.9; MS(MALDI-TOF): $m / z=853\left([M+N a]^{+}, 100 \%\right), 830\left(M^{+}, 50 \%\right) 653$ (10\%), 569 (50\%); HiRes-FT-MALDI-MS $\mathrm{m} / \mathrm{z}=830.4566$, calcd for $\mathrm{C}_{56} \mathrm{H}_{62} \mathrm{O}_{6}$ : 830.4541

[2]Rotaxane 1•4PF . The MPTTF-NP dumbbell $18(80 \mathrm{mg}, 0.060 \mathrm{mmol}), 20 \bullet 2 \mathrm{PF}_{6}$ (126 mg, $0.018 \mathrm{mmol}$ ), and dibromide 21 (47 $\mathrm{mg}, 0.018 \mathrm{mmol}$ ) were dissolved in anhydrous DMF (6 mL). The yellow reaction mixture was subjected to $10 \mathrm{kBar}$ at room temperature for $3 \mathrm{~d}$. The intense green solution was subjected directly to column chromatography $\left(\mathrm{SiO}_{2}\right)$. Unreacted dumbbell 18 was eluted with $\mathrm{Me}_{2} \mathrm{CO}$, whereupon the eluent was changed to $\mathrm{Me}_{2} \mathrm{CO} / \mathrm{NH}_{4} \mathrm{PF}_{6}(100: 1 \mathrm{v} / \mathrm{w})$ and the green band was collected. Most of the solvent was removed in vacuo followed by addition of $\mathrm{H}_{2} \mathrm{O}(25 \mathrm{~mL})$. The resulting precipitate was collected by filtration affording a green solid consisting of a mixture of the desired [2]rotaxane $1 \mathbf{1}^{-} \mathrm{PF}_{6}$ and the related [3] rotaxane (104 $\mathrm{mg}$ ). Separation by PTLC using $\mathrm{Me}_{2} \mathrm{CO} / \mathrm{NH}_{4} \mathrm{PF}_{6}(100: 1 \mathrm{v} / \mathrm{w})$ as the eluent afforded the pure compound $1.4 \mathrm{PF}_{6}\left(35 \mathrm{mg}, 23 \%\right.$ yield) as a green solid, $\mathrm{mp}>230{ }^{\circ} \mathrm{C}:{ }^{1} \mathrm{H} \mathrm{NMR}$ $\left(\left(\mathrm{CD}_{3}\right)_{2} \mathrm{CO}, 600 \mathrm{MHz}, 273 \mathrm{~K}\right.$, Green $): \delta=1.14(\mathrm{~d}, J=6.9 \mathrm{~Hz}, 12 \mathrm{H}), 1.26(\mathrm{~s}, 27 \mathrm{H}), 3.31$ (t, $J=6.0 \mathrm{~Hz}, 2 \mathrm{H}), 3.48$ (m, 2 H), 3.97 (m, 4 H), 4.01 (m, 4 H), 4.23 (m, 2 H), 4.45 (m, $2 \mathrm{H}), 6.05(\mathrm{~d}, J=13.8 \mathrm{~Hz}, 4 \mathrm{H}), 6.10(\mathrm{~d}, J=13.8 \mathrm{~Hz}, 4 \mathrm{H}), 6.44(\mathrm{~s}, 1 \mathrm{H}), 6.83$ (d, 
$J=8.9 \mathrm{~Hz}, 2 \mathrm{H}), 7.10(\mathrm{~m}, 11 \mathrm{H}), 7.14(\mathrm{~d}, J=7.8 \mathrm{~Hz}, 1 \mathrm{H}), 7.30(\mathrm{~d}, J=8.6 \mathrm{~Hz}, 6 \mathrm{H}), 7.37$ (s, $2 \mathrm{H}), 7.57(\mathrm{t}, J=8.2 \mathrm{~Hz}, 1 \mathrm{H}), 7.62(\mathrm{t}, J=8.2 \mathrm{~Hz}, 1 \mathrm{H}), 7.74(\mathrm{~d}, J=8.5 \mathrm{~Hz}, 2 \mathrm{H}), 7.89$ (t and d, $J=6.5$ and $8.5 \mathrm{~Hz}, 3 \mathrm{H}), 8.09(\mathrm{~m}, 9 \mathrm{H}), 8.39(\mathrm{~d}, J=7.2 \mathrm{~Hz}, 1 \mathrm{H}), 8.46(\mathrm{~d}, J=$ $6.4 \mathrm{~Hz}, 4 \mathrm{H}), 8.58$ (d, $J=6.4 \mathrm{~Hz}, 4 \mathrm{H}), 9.51(\mathrm{~d}, J=6.4 \mathrm{~Hz}, 4 \mathrm{H}), 9.62(\mathrm{~d}, J=6.4 \mathrm{~Hz}, 4$ $\mathrm{H}) ; \mathrm{MS}(\mathrm{ESI}): \mathrm{m} / \mathrm{z}=1075.0\left[\mathrm{M}-2 \mathrm{PF}_{6}\right]^{2+}, 668.3\left[\mathrm{M}-3 \mathrm{PF}_{6}\right]^{3+}, 561.2\left[19-\mathrm{PF}_{6}\right]^{+}, 465.0$ $\left[M-4 \mathrm{PF}_{6}\right]^{4+} .1 \bullet 4 \mathrm{PF}_{6}{ }^{2+}$ (Oxidation of 1•4 $\left.\mathrm{PF}_{6}\right):{ }^{1} \mathrm{H} \mathrm{NMR}\left(\left(\mathrm{CD}_{3}\right)_{2} \mathrm{CO}, 600 \mathrm{MHz}, 265 \mathrm{~K}\right.$, Red): $\delta=1.02(\mathrm{~d}, J=6.8 \mathrm{~Hz}, 12 \mathrm{H}), 1.24(\mathrm{~s}, 27 \mathrm{H}), 3.28(\mathrm{~m}, 2 \mathrm{H}), 3.46(\mathrm{~m}, 2 \mathrm{H}), 3.88(\mathrm{t}$, $J=5.1 \mathrm{~Hz}, 2 \mathrm{H}), 3.90$ (b, 2 H), 4.11 (b, $2 \mathrm{H}), 4.14$ (t, $J=5.1 \mathrm{~Hz}, 2 \mathrm{H}), 4.30$ (b, $2 \mathrm{H}), 4.55$ (b, 2 H), 4.65 (b, 2 H), 6.07 (d, $J=13.4 \mathrm{~Hz}, 4$ H), 6.14 (d, J = 13.4 Hz, 4 H), 6.18 (t, $J=7.9 \mathrm{~Hz}, 1 \mathrm{H}), 6.55(\mathrm{~m}, 2 \mathrm{H}), 6.81(\mathrm{~d}, J=8.9 \mathrm{~Hz}, 2 \mathrm{H}), 7.14(\mathrm{~m}, 3 \mathrm{H}), 7.22(\mathrm{~d}$, $J=8.6 \mathrm{~Hz}, 8 \mathrm{H}), 7.35(\mathrm{~d}, J=8.6 \mathrm{~Hz}, 6 \mathrm{H}), 7.39(\mathrm{~d}, J=7.2 \mathrm{~Hz}, 1 \mathrm{H}), 8.03(\mathrm{~d}, J=6.5 \mathrm{~Hz}$, $4 \mathrm{H}), 8.12$ (d, $J=6.51 \mathrm{~Hz}, 4 \mathrm{H}), 8.17$ (d, $J=8.4 \mathrm{~Hz}, 2 \mathrm{H}), 8.32$ (b, $8 \mathrm{H}), 8.45$ (d, $J=8.4$ Hz, 2 H), 8.80 (s, 2 H), 9.30 (d, J=6.4 Hz, 4 H), 9.51 (d, J=6.4 Hz, 4 H), 9.62 (s, $1 \mathrm{H})$.

[2]Rotaxane 2•4PF 6 . The NP-NP dumbbell 19 (50 mg, $0.060 \mathrm{mmol}), 20 \bullet 2 \mathrm{PF}_{6}(128 \mathrm{mg}$, $0.018 \mathrm{mmol}$ ) and 21 (48 $\mathrm{mg}, 0.018 \mathrm{mmol})$ were dissolved in anhydrous DMF (5 mL). The yellow reaction mixture was subjected to $10 \mathrm{kBar}$ at room temperature for $3 \mathrm{~d}$. The intense red solution was directly subjected to column chromatography $\left(\mathrm{SiO}_{2}\right)$. Unreacted dumbbell 19 was eluted with $\mathrm{Me}_{2} \mathrm{CO}$, whereupon the eluent was changed to $\mathrm{Me}_{2} \mathrm{CO} / \mathrm{NH}_{4} \mathrm{PF}_{6}(100: 1 \mathrm{v} / \mathrm{w})$ and the red band was collected. Removal of solvent in vacuo gave a dark red solution, followed by addition of $\mathrm{H}_{2} \mathrm{O}(25 \mathrm{~mL})$. The resulting red precipitate gave the desired [2] rotaxane $2 \bullet 4 \mathrm{PF}_{6}(9 \mathrm{mg}, 8 \%$ yield $), \mathrm{m} . \mathrm{p} .>230{ }^{\circ} \mathrm{C}:{ }^{1} \mathrm{H} \mathrm{NMR}$ $\left(\left(\mathrm{CD}_{3}\right)_{2} \mathrm{CO}, 600 \mathrm{MHz}, 298 \mathrm{~K}\right): \delta=1.12(\mathrm{~d}, J=6.9 \mathrm{~Hz}, 24 \mathrm{H}), 2.65(\mathrm{~b}, 4 \mathrm{H}), 3.45-3.53$ (m, $J=4.0 \mathrm{~Hz}, 4 \mathrm{H}), 4.14$ (b, 4 H), 4.21 (b, 4 H), 4.33 (b, 4 H), 4.58 (b, 4 H), 6.14 (s, 8 H), 6.92-7.13 (m, $14 \mathrm{H}), 8.00(\mathrm{~d}, J=6.0 \mathrm{~Hz}, 8 \mathrm{H}), 8.49(\mathrm{~s}, 8 \mathrm{H}), 9.38(\mathrm{~d}, J=6.0 \mathrm{~Hz}, 8$ H); $\mathrm{MS}(\mathrm{ESI}): \mathrm{m} / \mathrm{z}=830.3[\mathbf{1 9}]^{+}, 820.2\left[\mathrm{M}-2 \mathrm{PF}_{6}\right]^{2+}, 498.5\left[\mathrm{M}-3 \mathrm{PF}_{6}\right]^{3+}, 461.5,561.2$ $\left[20-\mathrm{PF}_{6}\right]^{+}, 337.6\left[M-4 \mathrm{PF}_{6}^{-}\right]^{4+}$. 
CHARACTERIZATION BY NMR SPECTROSCOPY: Figures S1 and S2 are the partial ${ }^{1} \mathrm{H}$ NMR spectra $\left(500 \mathrm{MHz},\left(\mathrm{CD}_{3}\right)_{2} \mathrm{CO}\right)$ of $1 \bullet 4 \mathrm{PF}_{6}$ at $198 \mathrm{~K}$ (low-field and highfield, respectively). These spectra show that even at low temperatures the $\mathrm{CBPQT}^{4+}$ ring encircles the MPTTF unit predominantly (see manuscript). Figures S3 and S4 are the COSY spectra $\left(600 \mathrm{MHz},\left(\mathrm{CD}_{3}\right)_{2} \mathrm{CO}\right)$ used to assign the ${ }^{1} \mathrm{H}$ NMR signals of $1 \bullet 4 \mathrm{PF}_{6}$ at $298 \mathrm{~K}$. The absence of scalar couplings between the high-field and low-field regions supports the presence of a single isomer predominantly in solution.

CHARACTERIZATION BY UV-VIS SPECTROSCOPY: Figures S6 and S7 show the effect that titration of $1 \bullet 4 \mathrm{PF}_{6}$ with one and two equiv. of $\mathrm{Fe}\left(\mathrm{ClO}_{4}\right)_{3}$, respectively, has on the absorption spectra. The absorptions of $\mathrm{MPTTF}^{\circ+}$ and $\mathrm{MPTTF}^{2+}$ dominate in Figures S6 and S7, respectively, making it impossible to speculate about the position of the $\mathrm{CBPQT}^{4+}$ ring. Figure S8 shows the effect on the UV-vis spectrum upon oxidation of the dumbbell compound $\mathbf{1 8}$.

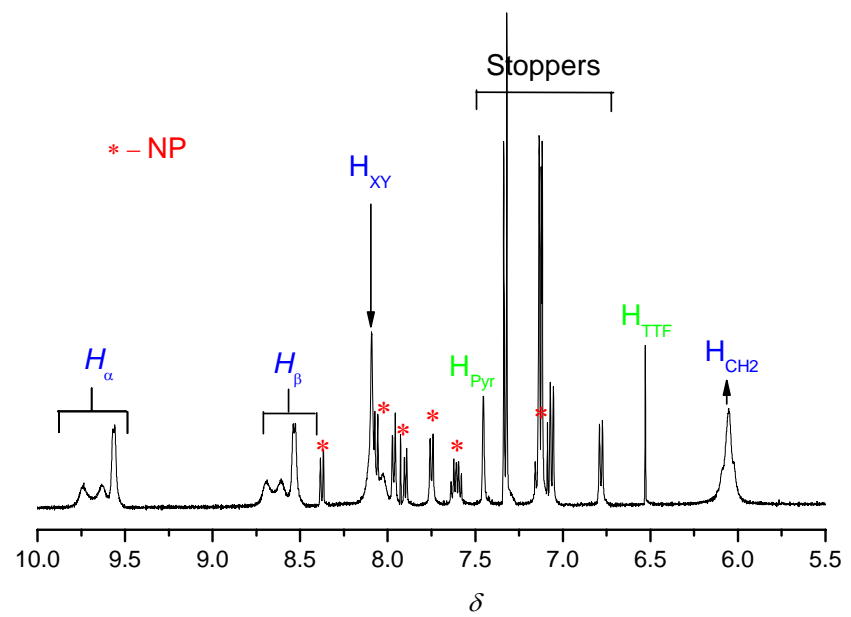

Figure S1. Partial ${ }^{1} \mathrm{H}$ NMR spectrum displaying the aromatic region $(500 \mathrm{MHz}$, $\left.\left(\mathrm{CD}_{3}\right)_{2} \mathrm{CO}\right)$ of $1.4 \mathrm{PF}_{6}$ at $198 \mathrm{~K}$. 


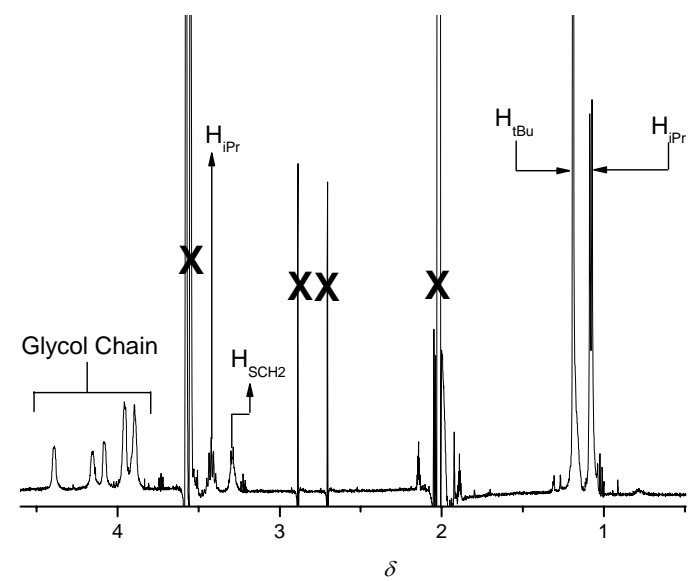

Figure S2. Partial ${ }^{1} \mathrm{H}$ NMR spectrum $\left(500 \mathrm{MHz},\left(\mathrm{CD}_{3}\right)_{2} \mathrm{CO}\right)$ displaying the aliphatic region of $1.4 \mathrm{PF}_{6}$ at $198 \mathrm{~K}$.

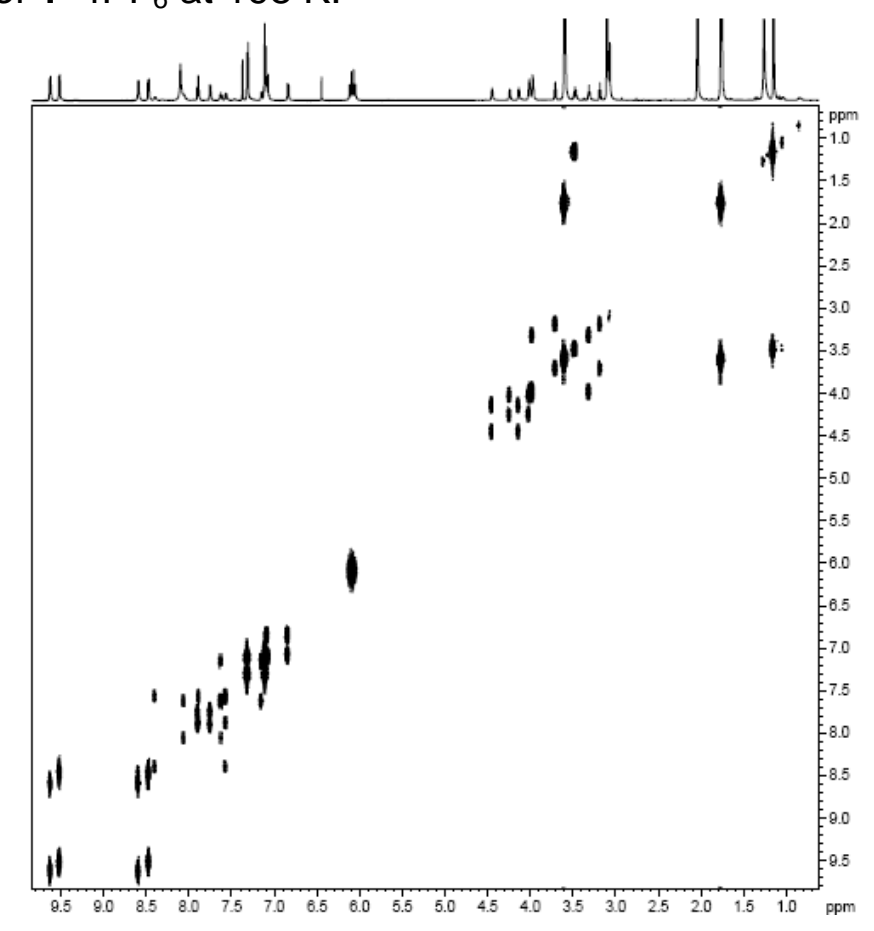

Figure S3. The full ${ }^{1} \mathrm{H}$ DQ COSY spectrum $\left(600 \mathrm{MHz},\left(\mathrm{CD}_{3}\right)_{2} \mathrm{CO}, 298 \mathrm{~K}\right)$ that was used to assign the proton signals of $1 \bullet 4 \mathrm{PF}_{6}$. 


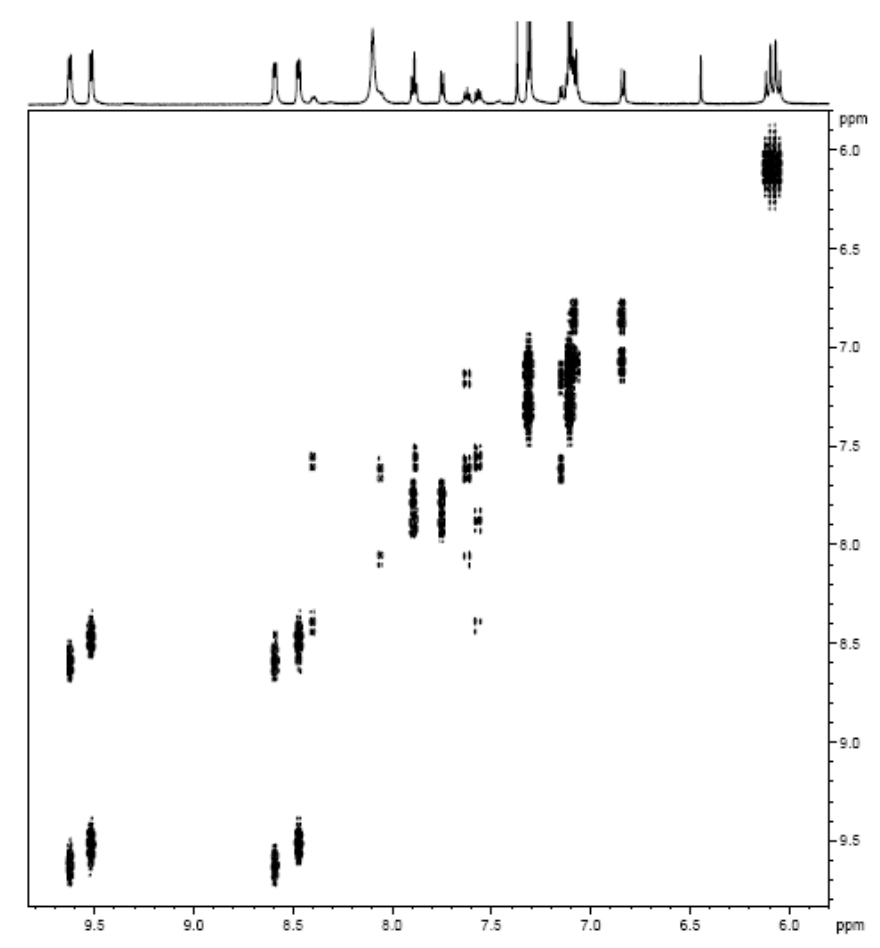

Figure S4. Zoom in on the low-field region of the ${ }^{1} \mathrm{H}$ DQ COSY spectrum $\left(600 \mathrm{MHz},\left(\mathrm{CD}_{3}\right)_{2} \mathrm{CO}, 298 \mathrm{~K}\right)$ of $1.4 \mathrm{PF}_{6}$.

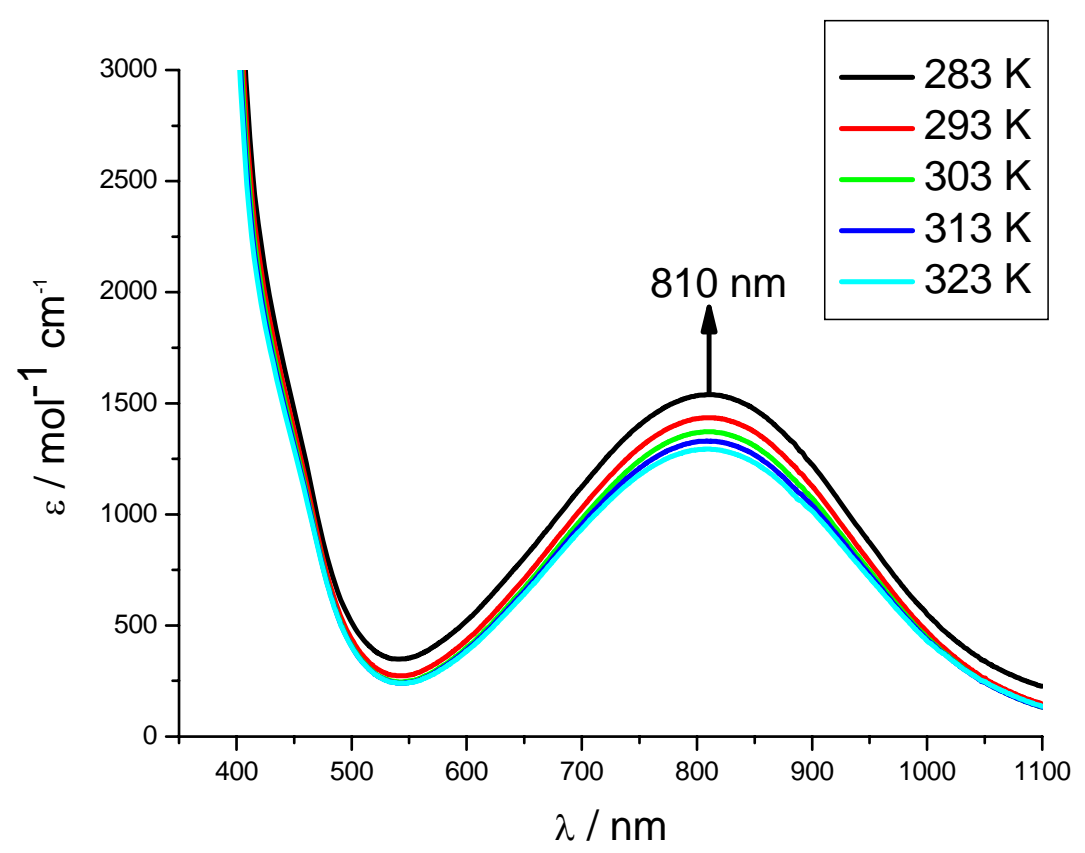

Figure S5. Absorption spectra of $1.4 \mathrm{PF}_{6}$ recorded in $\mathrm{MeCN}$ at various temperatures from 283 up to $323 \mathrm{~K}$. 


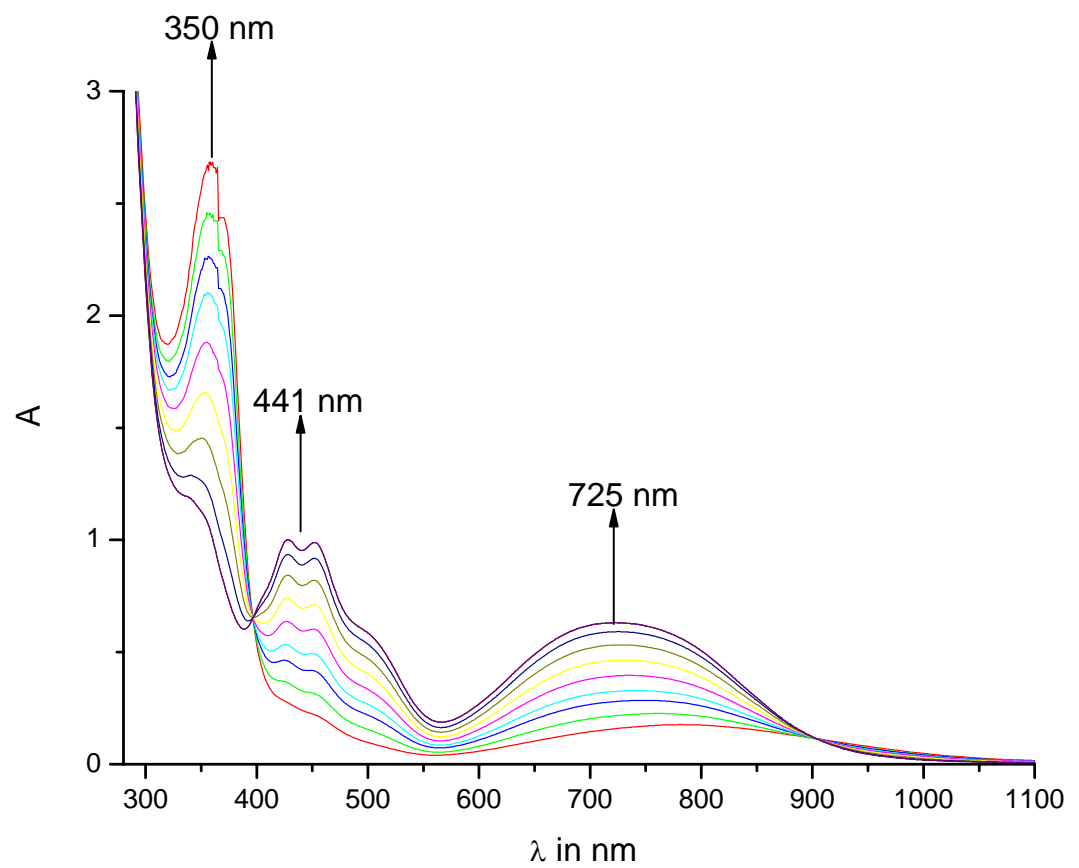

Figure S6. UV-vis spectra following the titrational addition of 1.0 equiv. of $\mathrm{Fe}\left(\mathrm{ClO}_{4}\right)_{3}$ to a solution of $1 \cdot 4 \mathrm{PF}_{6}$ in $\mathrm{MeCN}$.

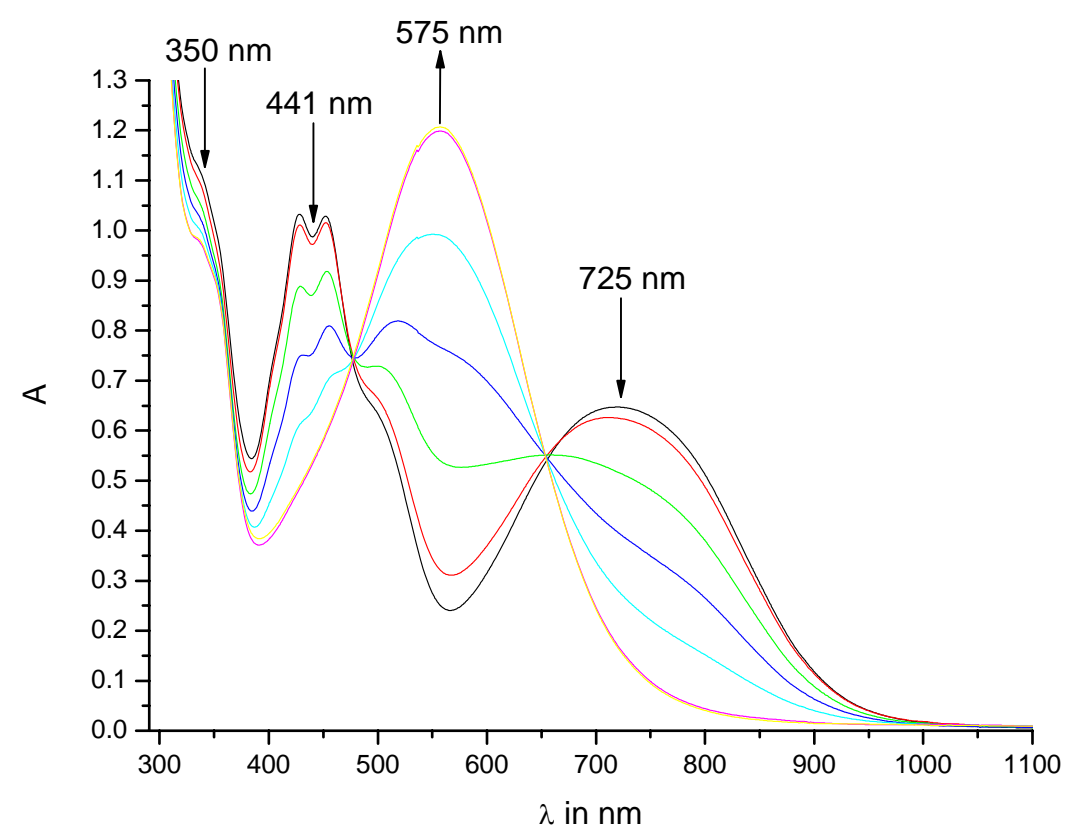

Figure S7. UV-vis spectra following the titrational addition of 1.0 equiv. of $\mathrm{Fe}\left(\mathrm{ClO}_{4}\right)_{3}$ to a solution of $\mathbf{1}^{5+}$ in $\mathrm{MeCN}$. 


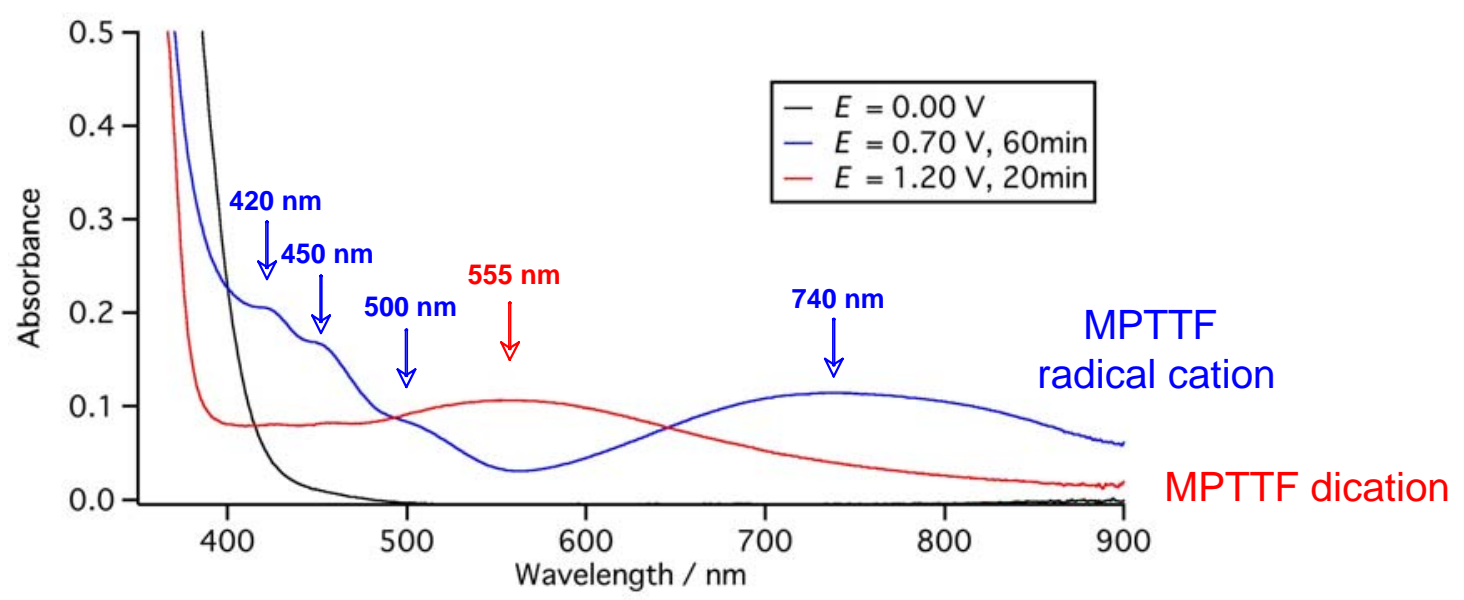

Figure S8. UV-vis spectra of the rigid MPTTF-NP dumbbell 18 upon oxidation. Condition: Argon purged THF at $298 \mathrm{~K}$; concentration $=0.25 \mathrm{mM}$; concentration of the electrolyte $\left(\mathrm{TBA} \cdot \mathrm{PF}_{6}\right)=0.1 \mathrm{M}$; working electrode = platinum net; counter electrode $=$ platinum coil; reference electrode $=$ silver wire .

Table S1. Electrochemical Data ${ }^{a}$ for the Model Compounds

\begin{tabular}{lcc}
\hline \multicolumn{1}{c}{ compound } & oxidation $^{b}$ & reduction $^{b}$ \\
\hline MPTTF half-dumbbell 10 & $+0.55,+0.75$ & - \\
NP half-dumbbell 17 & $+1.47^{c}$ & - \\
Rigid dumbbell 18 & $+0.55,+0.76,+1.41^{c}$ & - \\
CBPQT•4PF $_{6}$ & - & $-0.25,-0.70^{d}$ \\
\hline
\end{tabular}

\footnotetext{
${ }^{a}$ Argon-purged DMF, $1.0 \mathrm{mM}$ of the sample in $0.1 \mathrm{M} \mathrm{TBAPF}_{6}$ solution, glassy carbon as working electrode, r.t. ${ }^{b}$ Half-wave potential values in V vs SCE estimated from DPV peaks; reversible and monoelectronic processes, unless otherwise indicated. ${ }^{c}$ Irreversible process ${ }^{d}$ Two-electron reversible process.
}

\section{REFERENCES}

(1) Jeppesen, J. O.; Takimiya, K.; Jensen, F.; Brimert, T.; Nielsen, K.; Thorup, N.; Becher, J. J. Org. Chem. 2000, 65, 5794-5805. 
(2) Jia, C.; Zhang, D.; Guo, K.; Wan, S.; Zhu, D. Synthesis 2002, 2177-2182.

(3) Raehm, L.; Kern, J.-M.; Sauvage, J.-P. Chem. Eur. J. 1999, 5, 3310-3317.

(4) Anelli, P.-L.; Ashton, P. R.; Ballardini, R.; Balzani, V.; Delgado, M.; Gandolfi, M. T.; Goodnow, T. T.; Kaifer, A. E.; Philp, D.; Pietraszkiewicz, M.; Prodi, L.; Reddington, M. V.; Slawin, A. M. Z.; Spencer, N.; Stoddart, J. F.; Vicent, C.; Williams, D. J. J. Am. Chem. Soc. 1992, 114, 193-218.

(5) A. L. Van Geet, Anal. Chem. 1970, 42, 679-680.

(6) Attenburow, J.; Cameron, A. F. B.; Chapman, J. H.; Evans, R. M.; Hems, B. A.; Jansen, A. B. A.; Walker, T. J. J. Chem. Soc. Abs. 1952, 1104-1107.

\section{Complete References of the Main Text}

(6a) Anelli, P.-L.; Ashton, P. R.; Ballardini, R.; Balzani, V.; Delgado, M.; Gandolfi, M. T.; Goodnow, T. T.; Kaifer, A. E.; Philp, D.; Pietraszkiewicz, M.; Prodi, L.; Reddington, M. V.; Slawin, A. M. Z.; Spencer, N.; Stoddart, J. F.; Vicent, C.; Williams, D. J. J. Am. Chem. Soc. 1992, 114, 193-218. 